\title{
Statistical Analysis of Disease Reaction Data Using Nonparametric Methods
}

\author{
K.M. Eskridge ${ }^{1}$ \\ Department of Biometry, University of Nebraska, Lincoln, NE 68583-0712
}

Plant breeders developing disease-resistant horticultural crops need flexible and powerful statistical methods to compare treatments efficiently. The most commonly used statistical methods are those based on analysis of variance (ANOVA), regression, and correlation. The valid use of these methods requires that the data have 1) normally distributed responses, 2) constant error variance, 3) independently distributed errors, and 4) a correctly specified model. However, many plant disease experiments yield data that do not adhere to these standard assumptions. For example, in an experiment involving population levels of a pathogen, rarely will population levels be normally distributed. Field counts of the number of nondiseased individuals in a plot many times will have different variances for various treatments. Laboratory experiments measuring the amount of a particular chemical compound produced by a pathogen may have a few cultures with extremely high amounts of the compound, indicating the presence of outliers.

Using standard methods (e.g., ANOVA) on data such as these is questionable for several reasons. Any conclusions based on unreasonable assumptions are suspect and may be wrong simply because the chosen method of analysis forces assumptions on the data that cannot be justified. More importantly, use of inappropriate statistical methods many times reduces the chances of correctly identifying differences that would have been found had the correct method been used.

Nonparametric (NP) statistical methods can be useful alternatives to classical statistical methods. Nonparametric methods require fewer assumptions about the data, but in many cases, allow one to draw valid conclusions with considerably better chances of detecting differences between treatments. The objective of this paper is to demonstrate how nonparametric statistical methods can be simply applied to data likely to result from plant disease experiments.

\section{NONPARAMETRIC METHODS AND APPLICATIONS}

\section{ANOVA}

Many plant disease experiments involve measuring a quantitative response on each of several treatments. The main goal is to determine if responses differ among treatments. Some form of ANOVA is usually appropriate for testing equality of treatment effects. The specific type of ANOVA will depend on 1) the experiment design and 2 ) the distribution of the response variable. Nonparametric methods can be applied easily to several of the most commonly used designs, assuming the hypothesis of interest is no difference between true treatment medians. Use of medians is preferable to means when data are not normally distributed, because medians are less sensitive to extremes. However, when data are normal, the true mean equals the true median and statements about the medians apply to the means.

Completely randomized design (CRD). This type of experiment design consists of several experimental units being randomly assigned to each treatment, and a quantitative response is obtained from each unit. Nonparametric statistical methods are easily applied to data from a CRD. A simple approach is to use a nonparametric ANOVA based on ranks (Conover and Iman, 1981). The procedure is to 1) assign ranks

Received for publication 20 Oct. 1994. Accepted for publication 17 Nov. 1994 Published as paper no. 10792, Journal Series, Nebraska Agricultural Research Division, Univ. of Nebraska, Lincoln. The cost of publishing this paper was defrayed in part by the payment of page charges. Under postal regulations, this paper therefore must be hereby marked advertisement solely to indicate this fact.

${ }^{1}$ Associate Professor. to all data points; 2) using the classical CRD ANOVA, analyze the ranks as if they were the observed responses; and 3) if the F test is significant, conclude at least two treatment medians differ and use a multiple comparison method (e.g., LSD) on the ranked data to identify which treatments differ.

To illustrate the approach, data were taken from a study on the population levels of Xanthomonas campestris pv. phaseoli (Smith) Dye (Xcp) on dry beans (Phaseolus vulgaris L.) (Ishimaru et al., 1991). Xcp population levels were obtained on 10 leaves from each of two fields with different dry bean cultivars (Table 1). Population levels were skewed and nonnormally distributed $(P<0.012)$, and variances were extremely heterogeneous, severely violating assumptions of classical ANOVA. Nonparametric ANOVA on ranks revealed highly significant differences between the two fields $(\mathrm{F}=12.60 ; P=0.0023)$, while classical ANOVA indicated slight significance $(\mathrm{F}=5.87 ; P=$ 0.026). [The Appendix contains the necessary SAS statements to compute the nonparametric CRD ANOVA (SAS, 1992).] The nonparametric ANOVA appeared to have substantially more power because its $P$ value was more than 10 times smaller than that from the classical ANOVA.

Randomized complete block design (RCBD). This type of design is conducted so that experimental units are grouped into homogeneous blocks, with treatments being randomly assigned to units within each block and with a quantitative response obtained from each unit. The RCBD can be analyzed using nonparametric methods by 1) assigning ranks to data points within each block separately and 2) analyzing the ranks as if they are the observed responses using the classical RCBD ANOVA (Conover and Iman, 1981).

To demonstrate the use of this approach, data were taken from a study on the oxalic acid production of four strains of the fungus Sclerotinia sclerotiorum (Lib.) de Bary (Godoy et al., 1990; Table 2). One mycelium of each strain was prepared beginning at week 1, and the entire process was repeated for three more weeks, with each week serving as a block. Oxalic acid concentrations (milligrams per gram of dry weight of mycelium) were obtained for each mycelium. Variances differed extremely, with the ratio of the smallest to largest being $1.9 \times$ $10^{6}$. Use of classical ANOVA was not justified. Nonparametric ANOVA on the within-block ranks rejected equality of treatment medians at a high level of significance $(P<0.0001)$, while classical ANOVA showed only weak significance $(P=0.0478)$. (The Appendix contains the necessary SAS statements to compute a nonparametric RCBD ANOVA.) Comparison of the $P$ values apparently illustrated the improved power of the nonparametric approach. Use of the LSD on

Table 1. Population levels [colony forming units (CFU)] of foliar Xanthomonas campestris pv. phaseoli on dry beans from two fields (A and B), ranks, and field medians and variances (Ishimaru et al., 1991).

\begin{tabular}{|c|c|c|c|}
\hline \multicolumn{2}{|r|}{ Field A } & \multicolumn{2}{|c|}{ Field B } \\
\hline$\overline{\mathrm{CFU}}$ & Rank & $\mathrm{CFU}$ & Rank \\
\hline $\begin{array}{l}195,000 \\
\end{array}$ & 9 & 4,500 & 5 \\
\hline $1,810,000$ & 14 & $55,550,000$ & 19 \\
\hline 325,000 & 11 & $6,900,000$ & 15 \\
\hline 5,750 & 6 & $66,050,000$ & 20 \\
\hline 100 & 1 & $1,370,000$ & 12 \\
\hline 250,000 & 10 & $9,200,000$ & 16 \\
\hline 17,250 & 8 & $1,400,000$ & 13 \\
\hline 300 & 2 & 10,200 & 7 \\
\hline 2700 & 4 & $15,200,000$ & 17 \\
\hline 1700 & 3 & $34,550,000$ & 18 \\
\hline Median & 11,500 & & \\
\hline Variance & $3.1 \times 10^{11}$ & & \\
\hline
\end{tabular}


Table 2. Oxalic acid concentration (milligrams per gram) from four strains of Sclerotinia sclerotiorum mycelia for each of 4 weeks (blocks), ranks in parentheses, strain medians, and variances (Godoy et al., 1990).

\begin{tabular}{llccc}
\hline & \multicolumn{5}{c}{ Strain } \\
\cline { 2 - 5 } Week & \multicolumn{1}{c}{1} & \multicolumn{1}{c}{5} & 6 & 7 \\
\hline 1 & $2.40(4)$ & $0.23(3)$ & $0.00(1)$ & $0.01(2)$ \\
2 & $2.30(3)$ & $7.03(4)$ & $0.01(1)$ & $0.02(2)$ \\
3 & $5.01(3)$ & $26.93(4)$ & $0.02(2)$ & $0.01(1)$ \\
4 & $2.67(3)$ & $12.05(4)$ & $0.05(2)$ & $0.00(1)$ \\
Medians & $3.71 \mathrm{~A}^{z}$ & $9.54 \mathrm{~A}$ & $0.02 \mathrm{~B}$ & $0.02 \mathrm{~B}$ \\
Variance & 1.65 & 128.45 & $4.6 \times 10^{-4}$ & $6.6 \times 10^{-5}$ \\
\hline
\end{tabular}

${ }^{2}$ Two medians with the same letters do not differ at $\alpha=0.05$.

the ranked data revealed that strains 1 and 5 differed from strains 6 and $7(P<0.05)$, but no difference was detected between strains 1 and 5, nor between strains 6 and 7 (Table 2).

Analysis of other designs and treatment structures. Other types of experiment designs may be analyzed using similar ranking procedures. Koch (1970) demonstrated the use of nonparametric ANOVA for a split-split plot design. Conover (1980) and Conover and Iman (1981) discussed application of rank tests for other types of designs. Scheirer et al. (1976) presented rank methods for analyzing a factorial treatment structure.

\section{Correlation and regression analysis}

Many times, plant disease experiments involve measuring the strength of association between two quantitative variables. When both of these variables are normally distributed, estimates and tests are appropriately based on the classical Pearson product-moment correlation coefficient (Snedecor and Cochran, 1976). However, use of classical correlations is not justified when either one or both variables are far from normal. Spearman's rank correlation $\left(r_{s}\right)$ is a nonparametric correlation method that does not require any specific type of distribution and can be readily applied to non-normal quantitative variables (Conover, 1980). This approach proceeds by 1) assigning ranks to both variables individually and 2) computing Pearson's product-moment correlation coefficient based on these ranks.

Rank correlation analysis was used by Godoy et al. (1990) to measure the association between oxalic acid production and mycelia biomass of the fungus $S$. sclerotiorum (Table 3 ). Oxalic acid concentration was extremely non-normally distributed $(P<0.01)$, invalidating the use of classical correlation analysis. Rank correlation was appropriate and resulted in a correlation between oxalic acid and biomass of $\mathrm{r}_{\mathrm{s}}=+0.58$, which was significantly different from zero $(P$ $<0.02$ ). (The Appendix contains the necessary SAS statements to

Table 3. Oxalic acid (milligrams per gram) and mycelia biomass of the fungus Sclerotinia sclerotiorum, ranks, and rank correlation coefficient $\left(\mathrm{r}_{\mathrm{s}}\right)($ Godoy et al., 1990).

\begin{tabular}{lrrr}
\hline \hline & Oxalic acid & & \multicolumn{2}{c}{ Biomass } \\
\cline { 4 - 4 } Value & Rank & Value & Rank \\
\hline 2.40 & 11.0 & 0.14 & 1.0 \\
0.23 & 9.0 & 0.17 & 2.0 \\
0.00 & 1.5 & 0.26 & 6.5 \\
0.01 & 4.0 & 0.27 & 8.0 \\
2.30 & 10.0 & 0.45 & 14.0 \\
7.03 & 14.0 & 0.42 & 12.0 \\
0.01 & 4.0 & 0.21 & 4.0 \\
0.02 & 6.5 & 0.20 & 3.0 \\
5.01 & 13.0 & 0.47 & 15.0 \\
26.93 & 16.0 & 0.52 & 16.0 \\
0.02 & 6.5 & 0.31 & 9.5 \\
0.01 & 4.0 & 0.31 & 9.5 \\
2.67 & 12.0 & 0.41 & 11.0 \\
12.05 & 15.0 & 0.44 & 13.0 \\
0.05 & 8.0 & 0.26 & 6.5 \\
0.00 & 1.5 & 0.24 & 5.0 \\
Rank correlation $\mathrm{r}_{\mathrm{s}}=+0.58^{*}$ & &
\end{tabular}

*Significant at $\alpha=0.05$. compute a nonparametric rank correlation analysis.) This rank correlation showed a fairly strong association between oxalic acid and biomass and provided a valid $P$ value, whereas use of classical correlation and its $P$ value was clearly invalid due to non-normality. Note that, in the ranking of the variables, some data values were tied and the mean rank was assigned to the tied values (Table 3). For a further discussion of ties, consult Conover (1980).

Sometimes, plant disease experiments require use of simple or multiple regression. Theil (1950) presented a simple nonparametric method of estimating the slope and intercept for a simple linear regression. Conover (1980) discussed how to test for zero slope and how to use rank analysis with multiple regression.

\section{Categorical data analysis}

Categorical variables are common in plant disease experiments. Simple examples are: a seed is either viable or not; a line is either highly resistant, moderately resistant, or susceptible to a disease; a plant is one of four cultivars, etc. This type of data requires analyses different from the methods previously discussed because frequencies are analyzed instead of quantitative responses. When the dependent variable has I categories and there is one independent variable with $\mathrm{J}$ categories, data may be analyzed using contingency table analysis (Conover, 1980). The null hypothesis is that the probability of a particular response category $(\mathrm{i}=1, \ldots \mathrm{I})$ is homogeneous across the $\mathrm{J}$ treatments. This hypothesis is first tested using an overall chi-square statistic. The chi-square statistic $\left(\chi^{2}\right)$ is based on cell deviations of observed from expected frequencies, assuming $\mathrm{H}_{0}$ is true. Large deviations, and thus large chi-square values, tend to refute $\mathrm{H}_{0}$ because the expected values that are based on $\mathrm{H}_{0}$ are poor predictions of what was observed. When a large chi-square value rejects $\mathrm{H}_{0}$, the table may be subdivided into smaller tables, and chi-square analyses can be used to identify how the treatments differ.

To demonstrate the method, data from two resistant and two susceptible dry bean cultivars were evaluated for response to common bacterial blight pathogen (Aggour et al., 1989). About 30 seeds from each cultivar were spray-inoculated with Xcp directly before planting. Viable seedlings were then evaluated for symptoms (+ or - ) (Table 4). For cultivars with 30 seedlings, the expected number of seedlings with symptoms was $30 \times$ probability that a seed had symptoms. If the null hypothesis was true, then the estimated probability that a seed had symptoms was $38 / 121$, the total number of experimental seedlings with symptoms over the total number of seedlings. Thus, the expected number of seedlings with symptoms out of 30 was $30 \times(38 / 121)=$ 9.42. Similar reasoning gave the expected frequencies of symptomless seedlings. For a cell in the $2 \times 4$ table ( $I=2$ responses, $J=4$ cultivars; Table 4 ), the cell chi-square value was computed as the squared

Table 4. Frequency of seedlings of dry bean cultivars with or without common bacterial blight symptoms and chi-square values (Aggour et al., 1989).

\begin{tabular}{|c|c|c|}
\hline \multirow[b]{2}{*}{ Variable } & \multicolumn{2}{|c|}{ Symptoms } \\
\hline & Yes & No \\
\hline \multicolumn{3}{|l|}{ Cultivars } \\
\hline \multicolumn{3}{|l|}{ All, } \\
\hline Bac-6 & 2 & 29 \\
\hline V & 16 & 14 \\
\hline $\mathrm{PC}$ & 13 & 17 \\
\hline GNT & 7 & 23 \\
\hline \multicolumn{3}{|l|}{$\chi^{2} 18.546^{*}$} \\
\hline \multicolumn{3}{|l|}{ Resistant } \\
\hline Bac-6 & 2 & 29 \\
\hline GNT & 7 & 23 \\
\hline \multicolumn{3}{|l|}{$\chi^{2} 3.455$} \\
\hline \multicolumn{3}{|l|}{ Susceptible } \\
\hline V & 16 & 14 \\
\hline PC & 13 & 17 \\
\hline \multicolumn{3}{|l|}{$\chi^{2} \quad 0.602$} \\
\hline \multicolumn{3}{|l|}{ Bean type } \\
\hline Resistant & 9 & 52 \\
\hline Susceptible & 29 & 31 \\
\hline$\chi^{2} 15.832^{*}$ & & \\
\hline
\end{tabular}


deviation of observed from expected divided by expected. The overall chi-square statistic $\chi^{2}$ was the sum of all of the cell chi-square values. With these data, $\chi^{2}=18.546(\mathrm{df}=3)$, which was significant at $P<$ 0.001 . The data strongly refuted the null hypothesis of no difference among probabilities of symptoms across the four bean cultivars.

Although the overall chi-square strongly indicated differences among cultivars, it was not clear how the cultivars differed. Using the same ideas as with analyzing the $2 \times 4$ table, chi-square analysis was conducted on three $2 \times 2$ tables. One $2 \times 2$ table evaluated the differences among susceptible and resistant cultivars (Table $4 ; \chi^{2}=$ $15.832, \mathrm{df}=1, P<0.001)$. Another $2 \times 2$ table evaluated differences among the two resistant cultivars $\left(\chi^{2}=3.455, \mathrm{df}=1, P>0.05\right)$, while the third tested for differences between the two susceptible cultivars $\left(\chi^{2}=0.602\right.$, df $\left.=1, P>0.05\right)$. Based on these tables, the overall hypothesis was rejected due to resistant lines differing from the susceptible cultivars.

Other categorical response methods. Two-way contingency tables are restricted to a single treatment factor, which limits the usefulness of the method to simple experiments. However, many of the fundamental ideas of contingency tables extend to more than two dimensions and allow for the analysis of main effects and interactions of more complicated designs (Agresti, 1990; Freeman, 1987).

When the treatment variables are categorical and the response variable is binary, one approach is to use weighted least squares (Agresti, 1990). This method results in sums of squares that are appropriate for testing hypotheses of homogeneity of proportions for main effects and interactions in a manner similar to classical ANOVA. The idea of this approach is to obtain estimates that minimize the weighted error sums of squares where the weight of each proportion ( ) is the inverse of its variance $n_{i} /[(1-\quad)]$. These estimates then are used to obtain sums of squares for each of the relevant hypotheses. If a null hypothesis is true, the sums of squares has a chi-square distribution with the degrees of freedom from the relevant hypothesis. Appropriate use of the weight statement in SAS PROC GLM may be used to compute weighted sums of squares; however, SAS PROC CATMOD is somewhat easier to use because weights are automatically computed for each proportion (see Appendix).

I will use data from a study on the inheritance of common bacterial blight resistance in dry beans to illustrate the method (Arnaud-Santana et al., 1994). Seed from $64 \mathrm{~F}_{3} \mathrm{BC}_{2}$ recombinant inbred lines was obtained from two parental crosses (PC-50 x XAN-159, PC-50 x BAC-6), where PC-50 was a susceptible recurrent parent and the other two parents were resistant. Pods were inoculated with the common bacterial blight pathogen at the beginning of the pod suture. Seeds

Table 5. Number and proportion of diseased dry bean seeds for two locations (LOC) within the pod, two resistant parents (NR_PAR), and weighted least squares analysis of variance table (Arnaud-Santana et al., 1994)

\begin{tabular}{|c|c|c|c|}
\hline \multirow[b]{2}{*}{$\begin{array}{l}\text { Nonrecurrent } \\
\text { parent/response }\end{array}$} & \multicolumn{2}{|c|}{ Location } & \multirow[b]{2}{*}{ Total } \\
\hline & $\begin{array}{l}\text { Near } \\
\text { point }\end{array}$ & $\begin{array}{c}\text { Away from } \\
\text { point }\end{array}$ & \\
\hline \multicolumn{4}{|l|}{ BAC-6 } \\
\hline Proportion & 0.611 & 0.442 & 0.503 \\
\hline Number & 422 & 536 & 958 \\
\hline Total & 691 & 1214 & 1905 \\
\hline \multicolumn{4}{|l|}{ XAN159 } \\
\hline Proportion & 0.690 & 0.555 & 0.605 \\
\hline Number & 442 & 607 & 1049 \\
\hline Total & 641 & 1093 & 1734 \\
\hline \multicolumn{4}{|l|}{ Total } \\
\hline Proportion & 0.649 & 0.496 & 0.552 \\
\hline Number & 864 & 1143 & 2007 \\
\hline Total & 1332 & 2307 & 3639 \\
\hline Source & $\mathrm{df}$ & Chi-square & $P^{z}$ \\
\hline Intercept & 1 & $\overline{4766.48}$ & 0.0000 \\
\hline NR_PAR & 1 & 33.53 & 0.0000 \\
\hline LOC & 1 & 83.14 & 0.0000 \\
\hline NR_PAR $\times$ LOC & 1 & 1.11 & 0.2929 \\
\hline
\end{tabular}

from these lines were classified either next to the point of inoculation (or not) and whether symptoms were present after plating. The weighted least squares approach was used where the treatment variables were nonrecurrent parent (NR_PAR) and location of the seed in the pod (LOC), while the response variable was the proportion of diseased seeds for each parent-location combination.

Parent type and location did not interact $(P>0.05)$ regarding proportion of diseased seeds, as indicated by a nonsignificant sums of squares (chi-square) for interaction (Table 5). However, the nonrecurrent parent and location main effects were highly significant $(P<$ $0.05)$. BAC-6 had a smaller proportion of diseased seeds than XAN159 and seeds next to the point of inoculation had a higher chance of expressing symptoms.

\section{DISCUSSION}

Nonparametric methods are useful for the analysis of data from plant disease experiments. These methods require fewer assumptions and are often more powerful than the classical methods (see Iman et al., 1984). Many of these methods are conceptually similar to the classical statistical methods, and their application is generally accessible to horticultural scientists not possessing a detailed knowledge of statistics. Results from nonparametric methods are reported in a manner similar to results from standard methods. For example, treatment medians are given instead of means, and Spearman's rather than Pearson's correlation is reported.

I need to emphasize that nonparametric methods do have assumptions, although many methods are insensitive to violation of these assumptions (Conover, 1980). CRD and RCBD rank tests require that the underlying distributions for the treatments differ only by their medians, if at all. The RCBD rank test further assumes there is no block $\times$ treatment interaction. The methods presented above all require "large" sample sizes to be valid. [See Conover (1980) for required sample sizes for the above tests.] However, with "small" sample sizes, "exact" tests that provide the exact $P$ value are available for most of these methods (Conover, 1980).

\section{Literature Cited}

Aggour, A.R., D.P. Coyne, A.K. Vidaver, and K.M. Eskridge. 1989. Transmission of the common blight pathogen in bean seed. J. Amer. Soc. Hort. Sci. 114:1002-1008.

Agresti, A. 1990. Categorical data analysis. Wiley, New York.

Arnaud-Santana, E., D.P. Coyne, K.M. Eskridge, and A.K. Vidaver. 1994. Inheritance; low correlations of leaf, pod, and seed reactions to common blight disease in common beans; and implications for selection. J. Amer. Soc. Hort. Sci. 119:116-121.

Conover, W.J. 1980. Practical nonparametric statistics. 2nd ed. Wiley, New York.

Conover, W.J. and R.L. Iman. 1981. Rank transformation as a bridge between parametric and nonparametric statistics. Amer. Stat. 33(3):124-129.

Freeman, D.H. 1987. Applied categorical data analysis. Marcel Dekker, New York.

Godoy, G., J.R. Steadman, M.B. Dickman, and R. Dam. 1990. Use of mutants to demonstrate the role of oxalic acid in pathogenicity of Sclerotinia sclerotiorum on Phaseolus vulgaris. Physiol. Mol. Plant Pathol. 37:179191.

Iman, R.L., S.C. Hora, and W.J. Conover. 1984. Comparison of asymptotically distribution-free procedures for the analysis of complete blocks. J. Amer. Stat. Assn. 79:674-685.

Ishimaru, C.A., K.M. Eskridge, and A.K. Vidaver. 1991. Distribution analyses of epiphytic populations of Xanthomonas campestris pv. phaseoli on dry beans. Phytopathology 81:262-268.

Koch, G.G. 1970. The use of non-parametric methods in the statistical analysis of a complex split plot experiment. Biometrics 26:105-128.

SAS Institute. 1992. SAS user's guide: Statistics. vols. 1 and 2. SAS Institute, Cary, N.C.

Scheirer, C.J., W.S. Ray, and N. Hare. 1976. The analysis of ranked data derived from completely randomized factorial designs. Biometrics 32:429434.

Snedecor, G.W. and W.G. Cochran. 1976. Statistical methods. 6th ed. Iowa State Univ. Press, Ames.

Theil, H. 1950. A rank invariant method of linear and polynomial regression analysis. Indagationes Mathematicae 12:85-91. 